\title{
Arbor
}

\section{Perspectiva histórica sobre la Calidad de la Atención Sanitaria: evolución, tendencias y métodos}

\section{Paz Rodríguez Pérez}

Arbor CLXX, 670 (Octubre 2001), 371-381 pp.

Tras un recorrido por la evolución del concepto de calidad en diversos países, en este artículo, la autora de este artículo se decanta par una apuesta inicial hacia la excelencia, aunque considerando la búsqueda de la innovación y la anticipación como las claves del éxito en el futuro de las organizaciones sanitarias.

En 1988 Arnold S. Relman publicaba en el New Enland Journal of Medicine un artículo titulado "Assessment and Accountability. The Third Revolution in Medical Care». En este trabajo el autor menciona tres grandes revoluciones en el sistema sanitario estadounidense entre los años que van desde la Segunda Guerra Mundial hasta finales de los 80, que representarían el umbral de la tercera revolución, o el inicio de la era de la evaluación y de la calidad de la atención sanitaria. Entre los años 1940 a 1950 representaron la expansión y el desarrollo tecnológico, la época en la que aparecieron los mejores hospitales y servicios sanitarios americanos. Pero este desarrollo y crecimiento del gasto se hizo insostenible y dejó paso a la llamada segunda revolución o era de la contención de los costes. La frustración de muchos profesionales sanitarios por la preocupación excesiva y en ocasiones ex- 
clusiva de los gestores sobre la vertiente economicista de la sanidad y a su vez la incapacidad de estos gestores para frenar el crecimiento ilimitado del gasto sanitario, hizo que a finales de los años 80 se impusiera la necesidad de evaluar la utilización de los recursos sanitarios en función de resultados. Se iniciaba con este concepto lo que otro autores como Ellwood llamaron la época del «outcomes management», no sería posible ninguna política sanitaria de contención de gasto sin evaluar los resultados de las diferentes alternativas de atención sanitaria. Los años 90 y siguientes han representado para Estados Unidos, tal y como predijo Relman, la era del desarrollo de los sistemas de evaluación e investigación sobre las resultados de los servicios sanitarios.

Haciendo un paralelismo con este trabajo, podríamos decir que la evolución de nuestro sistema sanitario ha sido similar a la del estadounidense, aunque con alguna dilación en el tiempo. Así el desarrollo tecnológico se situaría a partir de los años 60, con la aparición de los grandes centros sanitarios y las primeras Unidades de Cuidados Intensivos en nuestro país. La era de la contención del gasto, o al menos de la trasferencia de esta preocupación a los profesionales sanitarios, por el excesivo crecimiento del gasto y la búsqueda de nuevas formulas de atención sanitaria, como alternativa a la costosa hospitalización tradicional, se inicia a mediados de los años 80 y es en los primeros años de la década de los 90 cuando la preocupación por la evaluación y la mejora de la calidad se impone en nuestro medio.

Sin embargo, aceptando que es reciente el inicio de la evaluación y mejora de la calidad de la asistencia sanitaria, es obligado decir que en todos los sectores, y muy especialmente en el sanitario, la búsqueda de esta calidad asistencial ha sido siempre una constante en el quehacer diario de todos los que hoy trabajamos o han trabajado en este campo. Las discrepancias sobre el origen de la evaluación de la calidad de los cuidados, no tendrían cabida para nosotros y aunque hay autores que lo sitúan 90 años atrás otros se remontan en la historia hasta 3000 años a. c.. Estas diferencias no son importantes y únicamente son atribuibles a que muchos autores entienden que el origen de la calidad de los cuidados esta en el origen de las estructuras formales que inician la evaluación de dicha atención. Apoyando ambas hipótesis revisamos aquí los dos pasados, el pasado lejano y el pasado reciente que abarca el presente siglo.

\section{El lejano pasado}

La evaluación sistemática de la calidad de la atención no es algo nuevo. Ya Hammurabi en el año 2000 a. c. promulgaba en Babilonia 
Perspectiva histórica sobre la Calidad.....

el código que regulaba la atención médica que incluía las multas que estos debían de pagar por los malos resultados de sus cuidados; también 2000 a. c. en el Papiro de Egipto encontramos algunos de los primeros estándares referidos a la práctica médica. En las culturas orientales como la China encontramos documentos que datan del año 1000 a. c. donde se presenta, exhaustivamente desarrollado, el estado del arte de la Medicina en aquella época y se regulan las competencias de los profesionales. El tratado de Hipócrates de Cos, 500 años a. c., recoge las primeras bases éticas y legales de obligado cumplimiento para los médicos y que aún hoy siguen vigentes. Más cercano en el tiempo y a nuestro medio no deben ser olvidados ni el tratado de Galeno (200 d. c.), que no es sino un gran trabajo donde se estandariza el conocimiento médico de la época, ni los trabajos que ya en 1600 publicó Vesalio en el campo de la Anatomía Humana.

Si hasta aquí hemos constatado básicamente estudios dirigidos a normalizar el proceso de la práctica clínica, los primeros trabajos de evaluación sistemática de dicha práctica comienzan a ser realizados por epidemiólogos. Así, entre estos trabajos que tratan de evaluar y conocer los resultados de la atención sanitaria, cabe mencionar el tratado sobre "Política Aritmética» que en el Siglo XVII escribía Sir W. Petty, uno de los padres de la epidemiología moderna, donde comparando los hospitales de Londres con los de París llega a afirmaciones como que «los hospitales de Londres son.... mejores que los de París, pues en los mejores hospitales de París fallecen 2 de cada 15 pacientes, mientras que en los peores hospitales de Londres fallecen 2 de cada 16 ..». No podemos dejar de mencionar los trabajos de Sir. W. Fahr y F. Nigtinghale a finales del siglo pasado sobre la mortalidad hospitalaria. Esta última fue una enfermera inglesa, que en 1855, analizando la mortalidad de los soldados procedentes de la Guerra de Crimea, y que fallecían tras amputarsele una pierna, observó que los que habían sido intervenidos en los grandes hospitales tenían mayor probabilidad de morir que los que habían sido operados en los hospitales pequeños. La principal causa de muerte era la infección intrahospitalaria, más frecuente en los grandes centros.

Tras estas experiencias puntuales de evaluación de la atención sanitaria, los primeros años del Siglo XX dan inicio a la evaluación sistemática de la calidad de la atención sanitaria y abre el período de lo que se ha venido a denominar como el pasado reciente.

\section{El pasado reciente y la realidad de nuestros días: el siglo XX}

En la Tabla 1 exponemos, por orden cronológico, aquellos hechos y/o personas que en el área de evaluación de la calidad de la asistencia 


\section{Paz Rodríguez Pérez}

Tabla 1

Hechos relevantes en la evolución de la evaluación de la calidad de la atención sanitaria:

1912.- La deficiente situación que presentaban los hospitales en los Estados Unidos a principios de siglo (falta de servicios centrales, mala organización,...) llevó a la recién surgida asociación de cirujanos (American College of Surgeons (ACS)) a retomar la iniciativa de Codman, cirujano del Massachusets General Hospital, que perdió su puesto de trabajo por intentar que en la atención de todos los pacientes se exigiera el cumplimiento de una serie de estándares mínimos: «Programa de estandarización de los hospitales».

1951.- El American College of Surgeons se fusiona con el American College of Physicians, la American Hospital Association, La American Medical Association y la Canadá Medical Association constituyendo la conocida y cada vez más activa Joint Commission on Acreditation of Hospital (JCAH). Años más tarde la Canadá Medical Association se separa para crear junto con otras asociaciones canadienses el Canadá Council on Hospital Acreditation difundiendo la acreditación de hospitales en Canadá.

1966.- Un hito en la historia del control de Calidad surge con Avedis Donabedian, quien, como ya hemos mencionado, formuló la ya conocida estrategia sobre la evaluación de la calidad asistencial basada en la estructura, el proceso y los resultados.

1972.- Surge en Estados Unidos la Professional Standards Review Organizations (PSRO) con el objetivo de revisar la calidad de la asistencia que prestaban los hospitales concertados con los programas MEDICARE y MEDICAID.

1973.- J. Wennberg inicia sus primeros trabajos sobre las variaciones de la práctica clínica entre diferentes áreas geográficas de Estados Unidos.

1974.- Aparece la Australian Council on Hospital Standars como una asociación de carácter gubernamental con el fin de realizar la acreditación de sus hospitales. Hoy es responsable de la publicación internacional Australian Quality Review (A.Q.R).

1978.- J. Williamson publica ?Assessing and Improving Health Care Outcomes: The Health Accounting Aproach to Quality Assurance?, un importante trabajo sobre las evaluación y mejora de los resultados de los servicios sanitarios.

1979.- Se crea en Holanda la CBO, organización para la asesoría de hospitales en temas de calidad y educación. Con fines no lucrativos, y aunque de ámbito preferentemente nacional, tiene también difusión internacional como lo refleja el que se encargue de publicar la European Newsletter on Quality Assurance.

1980.- Objetivo 31 de Salud para todos en el año 2.000 para la Oficina Regional Europea de la Organización Mundial de la Salud: «De aquí a 1.990, todos los Estados miembros deberán haber creado unas comisiones eficaces que aseguren la calidad de las atenciones a los enfermos en el marco de sus sistemas de prestaciones sanitarias». "Se podrá atender este objetivo si se establecen métodos de vigilancia, continúa y sistemática, para determinar la calidad de los cuidados prestados a los enfermos, convirtiendo las actividades de evaluación y control en una preocupación constante de las actividades habituales de los profesionales sanitarios, y finalmente, impartiendo a todo el personal sanitario una formación que asegure y amplíe sus conocimientos».

1988.- P. Ellwood propone el desarrollo de un programa nacional para Estados Unidos basado en orientar los resultados de la asistencia sanitaria (Outcomes Management) hacia el diseño de estándares y guías de práctica clínica.

1989.- D. Berwick propugna el Continuous Quality Improvement (CQI) como un modelo de mejora de la asistencia sanitaria, extrapolado del modelo industrial, válido para los servicios sanitarios.

1990.- La Joint Commission impulsa con la llamada agenda para el cambio la incorporación de los modelos de CQI y la necesidad de orientar los programas de evaluación hacia las la evaluación de resultados y a implicar a todos los profesionales en los proyectos de mejora. 
sanitaria han tenido un mayor impacto e influencia a lo largo del presente siglo (Tabla 1).

A continuación presentamos un análisis de los diferentes modelos y estrategias de los programas de evaluación de la atención sanitaria con especial referencia a la evolución en Estados Unidos.

Diferentes modelos y estrategias de los programas dirigidos a la evaluación y mejora de la calidad asistencial

Hasta mediados del Siglo XX una gran parte de las acciones de evaluación surgían de la iniciativa particular de los profesionales de la medicina, epidemiólogos o asociaciones científicas. Es esta etapa la que se corresponde con el modelo que $\mathrm{H}$. Palmer denomina como "profesional» y que se caracterizó básicamente por centrarse en la evaluación de las estructuras de los servicios y algo en resultados, por apenas disponer de criterios explícitos y donde el médico era considerado como «médico» y el paciente como «paciente» en el sentido más clásico de estos términos (Tabla 2). Es también en los últimos años del pasado siglo y los primeros de este cuando se inicia el período del llamado control activo de la calidad de los servicios sanitarios. Es cuando el sector industrial, tras la Revolución Industrial, inicia el paso del control pasivo al control activo de la calidad de sus productos, del control del consumidor, al control realizado por el productor. Hasta esas fechas, los consumidores cuando adquirían un producto que resultaba ser deficiente en alguna de sus características, exigían ser compensados por ello. Se hacía así un control pasivo de la calidad de dichos productos. Paulatinamente la industria fue asumiendo esta función de comprobar la calidad, tanto de la materia prima, como del proceso de producción $\mathrm{y}$ del producto final antes de que este fuera lanzado al mercado. Los productores se habían dado cuenta de que cuanto más irreversible y costoso era el proceso de producción más empeño había que poner, no en examinar la calidad de los productos, sino en construirlos con ella. En el sector Servicios y en especial en los servicios sanitarios, donde los errores son irreparables, esta iniciativa surge desde los profesionales de la Medicina.

Hacia los 60-70 la evaluación de la asistencia sanitaria comienza a ser no sólo importante para los profesionales sino que comienzan a interesarse los gobiernos y financiadores. De esta forma se inicia el período «burocrático», en el que las decisiones de los profesionales empiezan a estar marcadas por las reglas de la organización. Durante estos años comienza a producirse un cambio desde el análisis individual 


\section{Paz Rodríguez Pérez}

Tabla 2

Diferentes modelos de los programas de evaluación y mejora de la calidad asistencial

\begin{tabular}{|c|c|c|c|}
\hline Tipo de datos & PROFESIONAL & BUROCRÁTICO & PARTICIPATIVO \\
\hline Tipo de medidas & $\begin{array}{c}\text { estructura } \\
\text { resultados }\end{array}$ & $\begin{array}{c}\text { proceso } \\
\text { estructura }\end{array}$ & $\begin{array}{c}\text { adecuación } \\
\text { excelencia técnica } \\
\text { satisfacción }\end{array}$ \\
\hline $\begin{array}{c}\text { Concepto } \\
\text { - Médico } \\
- \text { Paciente }\end{array}$ & médico & $\begin{array}{c}\text { criterios explícitos: } \\
\text { sucesos centinela y técnicos }\end{array}$ & $\begin{array}{c}\text { criterios explícitos: } \\
\text { indic.aj, severidad, } \\
\text { calidad de vida }\end{array}$ \\
\hline
\end{tabular}

de patrones de cuidados hacia el análisis estadístico de los procesos. $\mathrm{Si}$ en un principio se caracteriza este período por ciertos matices de inspección, la transición de la inspección al análisis estadístico se produce en pocos años. Pero la transición desde la aproximación burocrática de los cuidados tuvo un escaso impacto, especialmente en los profesionales de la Medicina. Una estrecha definición de la calidad de los cuidados, que sólo pretendía buscar la conformidad con unos estándares y el énfasis en la información clínica, dejando en parte de lado a los profesionales de la atención sanitaria, era demasiado limitada e incapaz de mejorar la misma. La búsqueda de un equilibrio entre esa evaluación de la práctica clínica y la necesidad de considerar los servicios de salud, especialmente hospitales, como organizaciones complejas que necesitaban rediseñar sus procesos implicando a todos los que en ellos participaban, se imponía.

El modelo profesional había sido el primero en existir y había tenido una buena acogida por parte de los médicos. Los estándares profesionales eran revisados por pares y los mecanismos de control de la calidad eran responsabilidad de cada profesional. Hacia los años 70 el rápido desarrollo tecnológico de los servicios sanitarios y la variedad de complejas técnicas que iban emergiendo (UVI,..), el trabajo en equipo y la aparición de otros profesionales no médicos en los servicios sanitarios (terapeutas, técnicos..) hizo que la comunicación y la coordinación entre todos estos profesionales fueran motivos suficientes para la aparición del modelo burocrático, totalmente opuesto al profesionalizado. Contradicciones de este modelo organizacional son los requerimientos, por un lado de solicitar que las organizaciones realicen su auditoría interna y por otro lado la obligatoriedad de revisión externa sobre la utilización 
de recursos que hace el desarrollo de la Professional Standards Review Organization (PSRO) para los programas Medicaire y Medicaid. Estas dos estrategias de auditoría externa e interna son causas del entonces y todavía actual rechazo y escepticismo de los médicos sobre los programas de mejora de calidad. Esta etapa burocrática deriva en los conocidos modelos de Quality Assurance (Tabla 3).

Tabla 3

Algunas características de los modelos del Quality Assurance $y$ del Continuous Quality Improvement

\begin{tabular}{|c|c|c|}
\hline CARACTERISTICA & QA (Quality Assurance) & CQI (Continuous Quality Improvement) \\
\hline Punto de vista & Reactivo & Proactivo \\
\hline Objetivo & Conseguir la norma & Atender necesidad cliente \\
\hline Medición & Esporádica & Continuas \\
\hline Dirección & Centralizada (Coordinación) & Descentralizada (gestión) \\
\hline Acción & Recomienda & Implanta \\
\hline
\end{tabular}

A mediados de los años 80 en Estados Unidos comienzan a introducirse el modelo «industrial» en los servicios de salud. Aquí el paciente pasa de ser un beneficiario a ser un cliente. Las actividades de garantía de calidad son absorbidas por una estrategia de mejora continua de la calidad, donde la calidad debe de ser también una preocupación de los lideres, de los directivos de la organización y enfocada siempre a la búsqueda de las perspectivas de los clientes o consumidores. Hacer confluir los interés del médico, de la organización y del paciente puede ser «política y económicamente irresistible y éticamente gratificante». Es esta la línea propuesta por el conocido modelo del que hablaremos más adelante Mejora Continua o Continuous Quality Improvement (CQI).

\section{Algunas consideraciones sobre futuras tendencias en las acciones de evaluación y mejora de la atención sanitaria}

Calidad, responsabilidad y costes son hoy los tópicos de los Sistemas de Salud cercanos al nuestro. Si las décadas de los años 1960-70 se caracterizaron por la expansión y el desarrollo científico y tecnológico, las décadas siguientes lo están siendo por la necesidad de contención de los costes. Estamos ahora iniciando la etapa en la que la necesidad de consenso y de evaluación en el campo sanitario parece inevitable y como decía A. Relman no será viable ninguna política de contención de costes si no va unida a la evaluación de resultados. 
Sin embargo, entre los profesionales sanitarios permanece un gran escepticismo sobre los programas de mejora de la calidad. Son varias las razones para ello. En primer lugar, los médicos no observan grandes diferencias entre la nueva "Quality improvement» y la garantía de calidad que continua en cierto modo acosándoles. Tradicionalmente el "Quality asssurance» ha puesto el énfasis en identificar acciones de revisión de los proceso de atención, y analizarlo por las agencias externas de acreditación o los Comités hospitalarios con escasa participación de estos profesionales. La segunda razón del escepticismo es la poca evidencia de que los programas de «quality assurance» hicieran algo para mejorar la calidad de la atención. La falta de datos documentando la efectividad de esos programas colabora en parte al frecuente rechazo de esos profesionales y su difícil colaboración cuando el coordinador del programa de calidad propone una nueva acción y rara vez han hallado resultados o respuesta de otros profesionales o de los directivos sobre los anteriores programas. Una tercera razón para justificar el escepticismo de los médicos es la sospecha de que tras estas estrategias de mejora solo se esconde el imperativo de reducir los costes. Por todo ello los médicos tienen una serie de preguntas en ocasiones justificadas: ¿Qué hay de nuevo en el enfoque actual de la mejora de la calidad?; ¿puede en alguna medida ese enfoque ayudar a la mejora de los cuidados? o ¿es simplemente un esfuerzo encubierto de los planificadores de los servicios sanitarios para reducir los costes?

\section{La nueva aproximación a la mejora de la calidad}

Desde la perspectiva del Quality Assurance la calidad de los cuidados puede ser vista como el estudio de la distribución de los determinantes de los cuidados de salud en una población o de un proceso clínico determinado. Cuando estos están determinados, una segunda fase, la fase operacional debe de iniciarse.

Datos epidemiológicos y clínicos son necesarios en la primera fase. La estadística, la epidemiología, la sociología, la informática, etc, que hoy resultan todavía poco conocidas para los médicos como lo fue la farmacologia o la fisiología hace 100 años dejaran de serlo y ayudara a que las usen en su día a día dirigidas a la evaluación. Es necesario el uso de estas técnicas para detectar oportunidades de mejora, errores previsibles, eliminar la sobreutilización de recursos y los daños innecesarios para mejorar la calidad de nuestros servicios.

Pero la evaluación en sí misma no supondrá ninguna mejora de la calidad de la atención sanitaria. Es imprescindible la segunda fase, 
la fase operacional o de puesta en marcha de la información resultante de la evaluación. Es necesario la participación de quienes toman decisiones, cambios de actitudes, rediseño de procesos y disponer de modelos a seguir. Contar con información válida y fiable y la participación de profesionales sanitarios, directivos y pacientes en la mejora de la calidad de los cuidados, será lo que inicie el período del Continuous Quality Improvement (CQI).

Implantar los sistemas de CQI llevará entre 1 y 5 años en cada centro. No elimina las tradicionales funciones de la QA, sino que las absorbe. Para predecir el futuro de CQI en los servicios sanitarios podríamos hacer una extrapolación de lo que esta sucediendo en el sector industrial. La literatura del sector industrial pone de manifiesto el creciente desarrollo de los modelos de Calidad Total (TQM) como una estrategia o modelo de gestión. La forma de entender el TQM correctamente pasa por considerar a las personas y el aprendizaje continuo como el mayor valor de la empresa. El desarrollo de este modelo lleva consigo la aplicación de técnicas como la reingeniería de procesos, el «benchmarking», el liderazgo etc. y define la Gestión de Calidad como un sistema de aprendizaje continuado, que consigue no sólo el desarrollo de la empresa y la mejora de la calidad sino el del propio individuo también.

Según Ellis y Whittington en los hospitales estadounidenses quedan aún restos del tradicional QA que impiden o limitan el paso hacia el modelo del CQI o «continuo esfuerzo de todos los miembros de la organización para cubrir las necesidades y expectativas del cliente». La satisfacción del cliente, la satisfacción del empleado y el impacto en la sociedad se consiguen mediante el liderazgo en política y estrategia del personal, recursos y procesos, que llevan finalmente a la excelencia en los resultados. El manual de la JCHO en 1992 introduce gran cantidad de términos, de herramientas y vocabulario que confunden a los profesionales y no hacen fácil el cambio desde el movimiento del QA al CQI. Sin embargo, más de 4000 hospitales en US han comenzado a adoptar el modelo de CQI de la JCHCO. Una encuesta nacional en US hace pensar que estos hospitales están mas confortables con este nuevo método, aunque también evidencia un cierto retraso de los médicos en implicarse.

En organizaciones complejas como son los servicios sanitarios, los modelos más globales de abordar la gestión de la calidad como el Malcom Baldridge National Quality Award en Estados Unidos o su adaptación en el Modelo Europeo de Calidad, entienden ésta como una estrategia de mejora continua en todas y cada una de las unidades 
de la organización, involucrando a todos los profesionales en éste proceso, orientando la organización simultáneamente a usuarios y profesionales y por último desarrollando metodologías para medir y cuantificar en lo posible la calidad de los procesos. En la Tabla 4 se resumen las principales áreas a desarrollar y evaluar que propone el Modelo Europeo.

Tabla 4

Modelo EFQM

\begin{tabular}{|l|}
\hline CRITERIOS AGENTES: \\
\hline $\begin{array}{l}\text { LIDERAZGO: Comportamiento de todos los directivos para guiar la organización hacia la mejora } \\
\text { continua }\end{array}$ \\
\hline $\begin{array}{l}\text { POLITICA Y ESTRATEGIA: Misión, valores, visión y dirección estratégica de la organización hacia } \\
\text { la formulación, aplicación de estrategias y consecución de los objetivos. }\end{array}$ \\
\hline $\begin{array}{l}\text { GESTION DE PERSONAL: Como utiliza la organización los conocimientos y el potencial de su personal } \\
\text { para mejorar continuamente la prestación de los servicios a los ciudadanos }\end{array}$ \\
\hline RECURSOS: Gestión, utilización y conservación de los recursos en la organización \\
\hline $\begin{array}{l}\text { PROCESOS: Gestión de todas las actividades. Como se identifican y revisan los procesos y si es } \\
\text { necesario se corrigen para asegurar la mejora continua en todas las actividades de la organización. }\end{array}$ \\
\hline CRITERIOS RESULTADOS \\
\hline SATISFACCION DEL CLIENTE: Satisfacción del receptor último de los servicios prestados \\
\hline SATISFACCION DEL PERSONAL: Satisfacción de todos los empleados de la organización \\
\hline $\begin{array}{l}\text { IMPACTO EN LA SOCIEDAD: Que consigue la administración para satisfacer las necesidades de } \\
\text { la sociedad en general. Percepción de la sociedad en general sobre el impacto de la organización. }\end{array}$ \\
\hline RESULTADOS: Resultados en la obtención de los objetivos establecidos \\
\hline
\end{tabular}

La excelencia, la innovación y la anticipación, son las tres claves del éxito en el futuro de las organizaciones. La excelencia para Berwick será la clave del éxito sólo durante pocos años, después será la puerta de entrada, el requisito previo para cualquier organización. $\mathrm{Ni}$ el control estadístico del proceso, ni la mejora continua, ni el «benchmarking», etc., serán suficientes para mejorar. Sólo la búsqueda y el aprendizaje continuo de toda la organización será la forma para seguir sobreviviendo.

\section{Bibliografía}

1 Blumenthal D. Quality of Care. What is it?. N Engl J Med 1996: 335-891.

2 BROOK RH; MC GlYNN FA: CleARY PD. Measuring Quality of Care. N Engl J Med 1996: 335-966; 70.

3 Chassin MR. Improving Quality of Care. N Engl J Med 1996: 335-1062; 3. 


\section{Perspectiva histórica sobre la Calidad.....}

4 BERwICK DM. Payment by capitation and the Quality of Care. Care.N Engl J Med 1996: 335-1227; 31 .

5 ReLman AS. Assessment and accountability: THe third revolution in medical care. N Engl J Med 1988; 319: 1220-1220.

6 EISENBERG JM. Physicians utilisation: The state of research about physician's practice patterns. Medical Care 1985; 23:461-483.

7 BROOK RH. Maintaining hospital Quality. The need for international cooperation. JAMA 1993; 270(8): 985-7.

8 CHASSIN MR; KoSECOFF J; PARK RE et al. Does inapporpriate use explain geographic variations in the use of health services? A study of three procedures. JAMA 1987; 258: 2533-2537.

9 WENNBERG JE; BARRY MJ. Outcomes research. Science. 1994. May 6; 264 (5160): 758-9.

10 BRoOK R; LOHR K. Efficacy, effectiveness, variations and quality. Boundarycrossing research. Medical Care 1985; 23:710-22.

11 Quality in Health Care. Theory, Application and Evolution. Edited by N. O. Graham. Maryland 1995.

12 RodRíguez, P. Calidad Asistencial: Marco Teórico y Desarrollo Histórico. Curso Monográfico sobre Calidad en la Asistencia Sanitaria. Universidad Menendez Pelayo. Editado por SKB, Madrid 1998.

13 Rodríguez, P. Calidad Asistencial en Gestión Hospitalaria: Temes JL; Pastor V; Diaz JL. 2a Ed. Díaz de Santos. Madrid 1997. 